\title{
openheart Distinct risk factors of atrial fibrillation in patients with and without coronary artery disease: a cross-sectional analysis of the BOREAS-CAG Registry data
}

Naoto Murakami, ${ }^{1}$ Masaya Tanno, ${ }^{1}$ Nobuaki Kokubu, ${ }^{1}$ Junichi Nishida, ${ }^{1}$ Nobutaka Nagano, ${ }^{1}$ Hirofumi Ohnishi, ${ }^{2}$ Hiroshi Akasaka, ${ }^{1}$ Takayuki Miki, ${ }^{1}$ Kazufumi Tsuchihashi, ${ }^{1,3}$ Tetsuji Miura $^{1}$

To cite: Murakami N, Tanno M, Kokubu N, et al. Distinct risk factors of atrial fibrillation in patients with and without coronary artery disease: a cross-sectional analysis of the BOREAS-CAG Registry data. Open Heart 2017;4:e000573.

doi:10.1136/openhrt-2016000573

- Additional material is available. To view please visit the journal online (http://dx. doi.org/10.1136/openhrt2016-000573).

Received 17 November 2016 Revised 6 December 2016 Accepted 18 December 2016

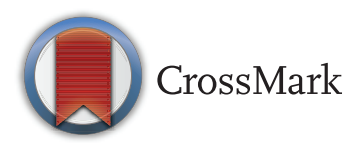

${ }^{1}$ Department of

Cardiovascular, Renal and Metabolic Medicine, Sapporo Medical University School of Medicine, Sapporo, Japan ${ }^{2}$ Department of Public Health, Sapporo Medical University School of Medicine, Sapporo, Japan

${ }^{3}$ Division of Health Care Administration and Management, Sapporo Medical University School of Medicine, Sapporo, Japan

Correspondence to Dr Masaya Tanno; tannom@sapmed.ac.jp

\section{ABSTRACT}

Objective: Although risk factors of atrial fibrillation $(\mathrm{AF})$ in the general population have been characterised, their impacts on patients with specific diseases are unclear. Our aim was to determine whether risk factors of $A F$ are different in patients with and those without coronary artery disease (CAD).

Methods: We enrolled 1871 consecutive patients who underwent coronary angiography for evaluation of symptoms suggestive of CAD in the BOREAS-CAG Registry between August 2014 and January 2015. After exclusion of patients with valvular heart disease or a history of $\mathrm{PCl} /$ cardiac surgery, 1150 patients contributed to multivariate logistic regression analysis to identify risk factors of AF. We also retrieved data for 361 consecutive patients with CAD admitted to Sapporo Medical University Hospital between April 2013 and July 2014 and analysed data for 166 patients using the same inclusion and exclusion criteria as those in the BOREAS-CAG Registry.

Results: Unexpectedly, CAD was independently associated with the absence of AF. The patients were then divided into a non-CAD group $(n=576)$ and a CAD group ( $n=574$ ) for further analysis. The brain natriuretic peptide level showed a strong association with $A F$ regardless of the presence or absence of CAD. In the non-CAD group, lack of statin use was independently associated with AF, whereas high serum uric acid level was an independent explanatory variable of $A F$ in the CAD group. The association of AF with uric acid was confirmed in a separate group of patients $(n=166)$ enrolled in the CAD cohort in Sapporo Medical University Hospital.

Conclusions: Major risk factors of $A F$ are different in patients with CAD and those without CAD. Patients with $\mathrm{CAD}$ are more likely to develop AF when the serum uric acid level is high, whereas no statin administration predicts development of $\mathrm{AF}$ in patients without CAD.

\section{INTRODUCTION}

Atrial fibrillation (AF) affects $1-2 \%$ of the general population and represents a rapidly

\section{KEY QUESTIONS}

What is already known about this subject?

- A number of cohort studies have shown clinical risk factors for the development of $A F$ in the general population, including advanced age, hypertension, diabetes and heart failure.

\section{What does this study add?}

- Major risk factors of AF in patients with certain clinical conditions may be different from those in the general population. No statin administration is associated with AF in patients without $C A D$, whereas a high uric acid level is a major risk factor associated with $A F$ in patients with CAD.

How might this impact on clinical practice?

- It needs to be confirmed by longitudinal studies whether uric acid-lowering agents and statins are effective for preventing the development of $A F$ in patients with $C A D$ and patients with cardiovascular risk factors but without $C A D$, respectively.

growing threat in ageing societies. Individuals with AF are at an approximately fivefold higher risk of stroke, ${ }^{2}$ sevenfold higher risk of heart failure and 1.9-fold higher risk of mortality ${ }^{3}$ than those without AF. AF plays a role in the development of heart failure with reduced ejection fraction (HFrEF) as well as heart failure with preserved ejection fraction ( $\mathrm{HFpEF}),{ }^{4}$ which has been becoming more and more prevalent in patients presenting with acute decompensated heart failure. ${ }^{5}$

Clinical risk factors for development of $\mathrm{AF}$ in the general population have been characterised by a number of cohort studies. ${ }^{6-8}$ Furthermore, simple score systems have been devised to calculate the risk of AF development by the use of variables such as age, 
race, body height, weight, blood pressure (BP), smoking status, antihypertensive medication, diabetes and history of myocardial infarction and heart failure. ${ }^{8-10}$ However, clinical factors that are not incorporated into the score systems also increase the risk of AF development under certain conditions. For example, it has been reported that renal dysfunction, ${ }^{11}$ presence of obstructive sleep apnoea $^{12}$ and right ventricular dysfunction ${ }^{13}$ were significant risk factors for the development of $\mathrm{AF}$ after cardiac surgery. Thus, it is unlikely that AF risk score systems are directly applicable to patients with morbidities, including coronary artery disease (CAD).

$\mathrm{CAD}$ is a risk factor of AF development as well as a disease for which the outcome is modulated by $\mathrm{AF}^{14-17}$ In a multicentre clinical study conducted in Denmark, ${ }^{16}$ the development of AF during index hospitalisation for MI was found to be associated with a significantly increased risk of sudden cardiac death. The presence of $\mathrm{CAD}$ in patients with previously diagnosed $\mathrm{AF}$ has been reported to be associated with recurrent $\mathrm{AF}$ episodes, ${ }^{17}$ symptomatic heart failure ${ }^{14}$ and increased mortality. ${ }^{18}$ However, it is not clear whether the presence of CAD simply adds a risk of AF or changes the impact of other risk factors of AF. To address this issue, we performed cross-sectional analyses to determine risk factors of AF in patients with and those without CAD by the use of data in the BOREAS (Broad-range Co-operative Organization for Renal, Arterial and Cardiac Studies by Sapporo Medical University Affiliates)-CAG Registry. We additionally performed similar analyses by the use of a separate database of patients with $\mathrm{CAD}$ to validate the findings in the BOREAS-CAG Registry patients.

\section{METHODS}

This study was conducted in strict accordance with the principles of the Declaration of Helsinki and was approved by the Clinical Investigation Ethics Committee of Sapporo Medical University Hospital. All of the study participants gave written informed consent.

\section{Study participants}

We retrieved data for 1871 consecutive patients registered in the BOREAS-CAG Registry from August 2014 to January 2015. The BOREAS-CAG Registry is a registry of consecutive patients who have undergone coronary angiography (CAG) in 12 affiliated hospitals. The indication for coronary angiography was in accordance with guidelines of the Japanese Circulation Society (JCS): guidelines for diagnostic evaluation of patients with chronic ischaemic heart disease, guidelines for management of acute coronary syndrome without persistent ST segment elevation and guidelines for the management of patients with ST-elevation acute myocardial infarction (MI). Patients with moderate or severe mitral valve disease or aortic valve disease and those with a history of percutaneous coronary intervention (PCI) and/or cardiac surgery including coronary artery bypass grafting
(CABG), aortic valve replacement, mitral valve replacement (MVR) and mitral valve plasty (MVP) were excluded, and the remaining 1150 patients aged 15-96 years contributed to the present analyses (figure 1). To confirm the findings in the BOREAS-CAG Registry participants, we also retrieved data for 361 consecutive patients with CAD admitted during the period from April 2013 to July 2014 from the database of the Department of Cardiovascular, Renal and Metabolic Medicine, Sapporo Medical University Hospital. This database consists of clinical data for patients who were admitted to the department and gave consent for providing their data to the database. The same exclusion criteria as those for the BOREAS-CAG Registry were applied after exclusion of patients with acute myocardial infarction, and the remaining 166 patients were included for the analyses. Demographic and clinical parameters were determined at the time of admission. CAD was defined as the presence of luminal diameter narrowing of $>50 \%$ in one or more major coronary arteries. The patients were assessed for the presence or absence of $\mathrm{AF}$ at a single time point, based on their 12-lead ECG at the time of enrolment or in the past or by Holter monitoring recorded in the past in some patients.

\section{Statistical analyses}

Numeric variables are expressed as means \pm SD for normal distributions or medians (IQRs) for skewed variables. The distribution of each parameter was tested for its normality using the Shapiro-Wilk W test, and nonnormally distributed parameters were logarithmically transformed for regression analyses. Differences between two groups were tested by Fisher's exact test or the $\chi^{2}$ test for categorical variables and by Student's $t$-test and the Kruskal-Wallis test for continuous variables with normal and skewed distributions, respectively. To assess the independent diagnostic values of the variables, we used multivariate stepwise logistic regression analyses based on Akaike's Information Criterion (AIC) for AF. A $\mathrm{p}$ value of $<0.05$ was considered significant. Statistical analyses were carried out using JMP (V.11 SAS Institute, Cary, North Carolina, USA).

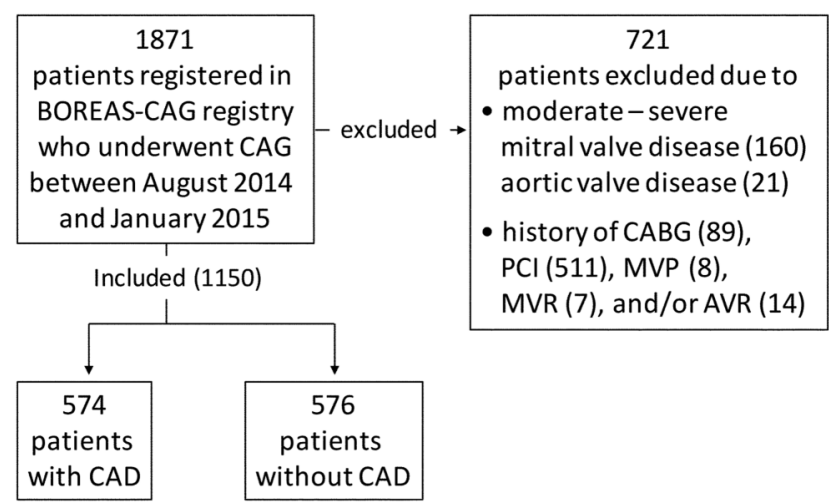

Figure 1 Flow chart summarising the study population. CAD, coronary artery disease. 


\section{RESULTS}

\section{Baseline characteristics of patients in the BOREAS-CAG Registry}

Clinical characteristics of the patients in the registry are shown in table 1.

Patients with AF were significantly older and less likely to have dyslipidemia than those without AF. The level of uric acid (UA) was higher, estimated glomerular filtration rate (eGFR) was lower, brain natriuretic peptide (BNP) level was higher and low-density lipoprotein (LDL) cholesterol level was lower in patients with AF than in those without AF. Statin and nicorandil were administered less often in patients with $\mathrm{AF}$ than in without $\mathrm{AF}$, whereas more patients with $\mathrm{AF}$ than patients without $\mathrm{AF}$ were treated with $\beta$ blockers, most likely to achieve optimal control of heart rate. Systolic BP was significantly lower in patients with $\mathrm{AF}$ than in patients without AF (131.7 $\pm 26.1 \mathrm{~mm} \mathrm{Hg}$ vs $143.8 \pm 29.0 \mathrm{~mm} \mathrm{Hg}, \mathrm{p}<0.001$ ), probably as a result of taking heart rate-lowering medications with negative inotropic actions, including $\beta$ blockers, diltiazem and/or verapamil. Thirty-five $(6.1 \%)$ of the 574 patients with $\mathrm{CAD}$ and $76(13.2 \%)$ of the 576 patients without $\mathrm{CAD}$ had AF (tables 1 and 3). Unexpectedly, the prevalence of CAD was significantly lower in patients with $\mathrm{AF}$ than in those without AF (table 1).

\section{Multivariate logistic regression analysis for AF in all enrolled participants}

Multivariate logistic regression analysis revealed that the presence of $\mathrm{AF}$ was independently associated with high BNP level, low systolic BP level and no statin administration in addition to the absence of CAD (table 2).
The association between $\mathrm{AF}$ and absence of $\mathrm{CAD}$ was unexpected since $\mathrm{CAD}$ is an established risk factor of $\mathrm{AF}^{7-10}$ However, the participants enrolled in this study were patients with an indication for coronary angiography, and the difference in entry criteria is likely to be an explanation for the discrepancy from the results of earlier studies in general populations. The independent association of low systolic BP level with $\mathrm{AF}$ was most likely due to the effect of drug therapy for AF.

\section{Baseline characteristics: non-CAD group versus CAD group}

We next examined differences in clinical characteristics and AF risk factors according to the presence $(\mathrm{n}=574)$ and absence $(\mathrm{n}=576)$ of CAD (figure 1 and online supplementary table $\mathrm{S} 1$ ).

As shown in online supplementary table S1, the patients in the $\mathrm{CAD}$ group had well-known coronary risk

Table 2 Multivariate logistic regression analysis for AF

\begin{tabular}{llll}
\hline & OR & 95\% Cl & p Value \\
\hline log BNP & 1.59 & 1.30 to 1.99 & $<0.001$ \\
CAD & 0.35 & 0.20 to 0.62 & $<0.001$ \\
Systolic BP (mm Hg) & 0.98 & 0.98 to 0.99 & 0.001 \\
Statin & 0.51 & 0.28 to 0.90 & 0.019 \\
$\beta$ blocker & 1.69 & 0.97 to 2.90 & 0.064 \\
Age (years) & 1.02 & 1.00 to 1.05 & 0.098 \\
DM & 0.68 & 0.38 to 1.18 & 0.171 \\
eGFR (mL/min/1.73m $\left.{ }^{2}\right)$ & 0.99 & 0.98 to 1.00 & 0.247 \\
Sex (male) & 0.91 & 0.53 to 1.59 & 0.747 \\
\hline
\end{tabular}

$\mathrm{AF}$, atrial fibrillation; $\mathrm{CAD}$, coronary artery disease;

eGFR, estimated glomerular filtration ratio.

Table 1 Baseline characteristics of patients in the BOREAS-CAG Registry

\begin{tabular}{|c|c|c|c|c|}
\hline & Overall $(n=1150)$ & AF $(n=111)$ & Non-AF $(n=1039)$ & p Value \\
\hline Age (years) & $68.8 \pm 12.0$ & $71.2 \pm 8.5$ & $68.5 \pm 12.3$ & 0.026 \\
\hline Sex (male) & $772(67.1)$ & $72(64.9)$ & $700(67.6)$ & 0.555 \\
\hline BMI $\left(\mathrm{kg} / \mathrm{m}^{2}\right)$ & $24.4 \pm 4.0$ & $24.5 \pm 4.3$ & $24.4 \pm 4.0$ & 0.795 \\
\hline CAD & $574(49.9)$ & 35 (31.5) & 539 (51.9) & $<0.001$ \\
\hline Smoking & $648(60.1)$ & $67(61.5)$ & $581(60.0)$ & 0.760 \\
\hline Systolic BP (mm Hg) & $142.6 \pm 29.0$ & $131.7 \pm 26.1$ & $143.8 \pm 29.0$ & $<0.001$ \\
\hline $\mathrm{HT}$ & $751(69.0)$ & $76(68.5)$ & $675(69.1)$ & 0.893 \\
\hline DM & 408 (37.6) & 35 (31.5) & 373 (38.3) & 0.161 \\
\hline $\mathrm{DL}$ & $582(53.9)$ & $45(40.5)$ & $537(55.4)$ & 0.003 \\
\hline UA (mg/dL) & $5.9 \pm 1.8$ & $6.4 \pm 2.0$ & $5.9 \pm 1.7$ & 0.002 \\
\hline eGFR $\left(\mathrm{mL} / \mathrm{min} / 1.73 \mathrm{~m}^{2}\right)$ & $65.5 \pm 30.0$ & $55.7 \pm 21.7$ & $66.6 \pm 30.6$ & $<0.001$ \\
\hline BNP $(p g / m L)$ & $48.0(18.6-131.5)$ & $148.0(69.3-496.0)$ & $42.4(17.0-105.0)$ & $<0.001^{*}$ \\
\hline LDL (mg/dL) & $107.5 \pm 32.8$ & $100.1 \pm 29.9$ & $108.3 \pm 33.0$ & 0.021 \\
\hline HbA1c (\%) & $6.3 \pm 1.3$ & $6.3 \pm 1.1$ & $6.3 \pm 1.3$ & 0.872 \\
\hline$\beta$ blocker & $273(25.8)$ & $50(45.5)$ & $223(23.6)$ & $<0.001$ \\
\hline ACEI/ARB & $471(44.4)$ & $54(49.1)$ & 417 (43.9) & 0.295 \\
\hline Statin & 472 (44.6) & $29(26.6)$ & $443(46.7)$ & $<0.001$ \\
\hline Nicorandil & 246 (23.3) & $16(14.8)$ & $230(24.3)$ & 0.028 \\
\hline
\end{tabular}


Table 3 Clinical characteristics of non-CAD and CAD groups with and without AF

\begin{tabular}{|c|c|c|c|c|c|c|}
\hline & \multicolumn{2}{|l|}{ Non-CAD group } & \multirow[b]{2}{*}{ p Value } & \multicolumn{2}{|l|}{ CAD group } & \multirow[b]{2}{*}{ p Value } \\
\hline & AF $(n=76)$ & Non-AF $(n=500)$ & & AF $(n=35)$ & Non-AF $(n=539)$ & \\
\hline Age (years) & $70.7 \pm 8.5$ & $67.6 \pm 13.1$ & 0.046 & $72.3 \pm 8.5$ & $69.4 \pm 11.5$ & 0.126 \\
\hline Sex (male) & $46(60.5)$ & 297 (59.5) & 0.868 & $26(74.3)$ & 403 (75.2) & 0.090 \\
\hline BMI $\left(\mathrm{kg} / \mathrm{m}^{2}\right)$ & $25.1 \pm 4.2$ & $24.6 \pm 4.3$ & 0.349 & $23.3 \pm 4.5$ & $24.3 \pm 3.7$ & 0.154 \\
\hline Smoking & $42(56.8)$ & $258(55.5)$ & 0.838 & $25(71.4)$ & $323(64.1)$ & 0.380 \\
\hline $\mathrm{HT}$ & $51(67.1)$ & 306 (65.7) & 0.806 & $25(71.4)$ & 369 (72.2) & 0.920 \\
\hline DM & 23 (30.3) & $157(33.8)$ & 0.540 & 12 (34.3) & $216(42.4)$ & 0.345 \\
\hline $\mathrm{DL}$ & $27(35.5)$ & $229(49.6)$ & 0.023 & $18(51.4)$ & $308(60.8)$ & 0.276 \\
\hline UA (mg/dL) & $6.3 \pm 1.6$ & $5.9 \pm 1.8$ & 0.054 & $6.7 \pm 2.7$ & $5.9 \pm 1.7$ & 0.007 \\
\hline $\begin{array}{l}\text { eGFR } \\
\left(\mathrm{mL} / \mathrm{min} / 1.73 \mathrm{~m}^{2}\right)\end{array}$ & $56.5 \pm 20.1$ & $68.7 \pm 23.8$ & $<0.001$ & $54.0 \pm 24.9$ & $64.7 \pm 35.6$ & 0.041 \\
\hline $\mathrm{BNP}(\mathrm{pg} / \mathrm{mL})$ & $144.3(74.4-437.3)$ & $33.8(14.5-92.1)$ & $<0.001^{*}$ & $168.4(52.8-687.9)$ & $49.0(19.3-123.1)$ & $<0.001^{*}$ \\
\hline LDL (mg/dL) & $98.3 \pm 30.9$ & $106.1 \pm 32.1$ & 0.072 & $103.9 \pm 27.6$ & $110.4 \pm 33.7$ & 0.315 \\
\hline $\mathrm{HbA1c}(\%)$ & $6.3 \pm 1.1$ & $6.2 \pm 1.2$ & 0.356 & $6.3 \pm 1.1$ & $6.4 \pm 1.4$ & 0.746 \\
\hline$\beta$ blocker & $36(48.0)$ & $102(22.7)$ & $<0.001$ & $14(40.0)$ & $121(24.3)$ & 0.039 \\
\hline ACEI/ARB & $36(48.0)$ & 193 (42.6) & 0.383 & $18(51.4)$ & $224(45.0)$ & 0.459 \\
\hline Statin & $15(20.3)$ & $187(41.5)$ & $<0.001$ & $14(40.0)$ & $256(51.4)$ & 0.192 \\
\hline Nicorandil & $7(9.6)$ & 69 (15.3) & 0.1987 & $9(25.7)$ & $161(32.4)$ & 0.413 \\
\hline
\end{tabular}

Data are given as the mean \pm SD or medians (IQRs), or number of patients with percentage in brackets.

${ }^{*}$ Kruskal-Wallis test.

ACEI/ARB, angiotensin-converting enzyme inhibitors/angiotensin II type 1 receptor blockers; AF, atrial fibrillation; BMI, body mass index; BNP, B-type natriuretic peptide; CAD, coronary artery

disease; DL, dyslipidemia; DM, diabetes mellitus; eGFR, estimated glomerular filtration ratio; HT, hypertension; LDL, low-density lipoprotein cholesterol; UA, uric acid. 
factors than did those in the non-CAD group; age, percentage of males and prevalences of current or past smoking, hypertension (HT), diabetes (DM) and dyslipidemia (DL) were all significantly higher in the CAD group than in the non-CAD group. Despite significantly more frequent administration of statins in the CAD group, the level of LDL cholesterol was still significantly higher in the CAD group. The CAD group showed significantly higher levels of HbAlc than those in the non-CAD group. eGFR was lower and BNP was higher in the CAD group than in the non-CAD group, but the differences did not reach statistical significance. There were no inter-group differences in the use of $\beta$ blockers and angiotensin-converting enzyme inhibitors (ACEI)/angiotensin type 1 receptor blockers (ARB). The prevalence of $\mathrm{AF}$ in patients in the BOREAS-CAG Registry (9.7\%) was much higher than that in the general population, ${ }^{1}$ in agreement with the high prevalences of risk factors including HT, DM and heart failure (table 1). When analysed separately (see online supplementary figure S1), the non-CAD group showed a significantly higher prevalence of either paroxysmal AF (PAF) or chronic AF $(\mathrm{CAF})$, as well as overall $\mathrm{AF}$, than did the $\mathrm{CAD}$ group (5.6\% vs $3.1 \%$ for PAF, $7.6 \%$ vs $3.0 \%$ for $\mathrm{CAF}$ and $13.2 \%$ vs $6.1 \%$ for overall $\mathrm{AF}$, all $\mathrm{p}<0.001)$. The percentages of patients who were administered a statin $(50.7 \%$ vs $38.5 \%$, $\mathrm{p}<0.001)$ and nicorandil (32.0\% vs $14.5 \%, \mathrm{p}<0.001)$ were significantly higher in the CAD group than in the non-CAD group (see online supplementary table S1).

\section{Clinical characteristics of patients with AF and those without $A F$ in the non-CAD group}

In the non-CAD group, patients with $\mathrm{AF}$ were significantly older and had significantly lower prevalence of DL, lower eGFR and higher BNP than patients without AF (table 3).

The percentage of patients who were administered a statin was much lower for patients with $\mathrm{AF}$ than for those without $\mathrm{AF}(20.3 \%$ vs $41.5 \%, \mathrm{p}<0.001$, table 3$)$. More patients with $\mathrm{AF}$ were taking $\beta$ blockers than were patients without $\mathrm{AF}$, as was the case with the overall data in this registry.

\section{Multivariate logistic regression analysis for $\mathrm{AF}$ in the non-CAD group}

Multivariate logistic regression analysis demonstrated that BNP was independently associated with the presence of AF $(p<0.001)$ and that statin administration was highly predictive of the absence of AF ( $p=0.006$, table 4) in the non-CAD group (table 4). The association of statin administration with the absence of $\mathrm{AF}$ suggests that a statin may suppress development of AF by reducing the LDL level. However, the level of LDL paradoxically tended to be higher in patients without AF than in those with $\mathrm{AF}(106.1 \pm 32.1 \mathrm{mg} / \mathrm{dL}$ vs $98.3 \pm 30.9 \mathrm{mg} / \mathrm{dL}$, $\mathrm{p}=0.072$, table 3 ) in the non-CAD group. Indeed, no treatment with a statin was associated with a significantly higher prevalence of $\mathrm{AF}$ regardless of the level of $\mathrm{LDL}$

\begin{tabular}{lccr}
\multicolumn{4}{l}{ Table 4 Multivariate logistic regression analysis for AF } \\
\hline \multicolumn{1}{l}{ OR } & $\mathbf{9 5 \%} \mathbf{C l}$ & p Value \\
\hline Non-CAD group & & & \\
log BNP & 1.84 & 1.41 to 2.43 & $<0.001$ \\
Statin & 0.36 & 0.16 to 0.76 & 0.006 \\
$\beta$ blocker & 1.87 & 0.94 to 3.70 & 0.075 \\
eGFR (mL/min/1.73 $\mathrm{m}^{2}$ ) & 0.99 & 0.97 to 1.01 & 0.146 \\
Age (years) & 1.01 & 0.99 to 1.04 & 0.328 \\
DM & 0.71 & 0.34 to 1.43 & 0.348 \\
Sex (male) & 1.12 & 0.58 to 2.15 & 0.744 \\
CAD group & & & \\
log BNP & 1.56 & 1.18 to 2.07 & $<0.001$ \\
UA (mg/dL) & 1.21 & 1.00 to 1.50 & 0.047 \\
DM & 0.52 & 0.20 to 1.26 & 0.151 \\
Age (years) & 1.02 & 0.98 to 1.07 & 0.428 \\
Sex (male) & 0.8 & 0.31 to 2.26 & 0.657 \\
\hline
\end{tabular}

$\mathrm{AF}$, atrial fibrillation; $\mathrm{CAD}$, coronary artery disease;

eGFR, estimated glomerular filtration ratio; UA, uric acid.

cholesterol in the non-CAD group (see online supplementary figure S2).

\section{Clinical characteristics of patients with AF and those without AF in the CAD group}

When the clinical characteristics of patients with $\mathrm{AF}$ and those without $\mathrm{AF}$ in the $\mathrm{CAD}$ group were compared, significantly higher UA, lower eGFR and higher BNP were found in patients with AF (table 3). Patients in the CAD group were administered similar medications regardless of the presence or absence of AF, though the percentage of patients prescribed a $\beta$ blocker was significantly higher for patients with $\mathrm{AF}$ than for those without $\mathrm{AF}$, as was the case in the non-CAD group.

\section{Multivariate logistic regression analysis for AF in the CAD group}

Multivariate logistic regression analysis showed that BNP and UA levels were independently associated with $\mathrm{AF}$ in patients with CAD (ORs: 1.56 per $\log \mathrm{BNP}$ and 1.21 per $\mathrm{mg} / \mathrm{dL}$ for UA, table 4 ).

\section{Clinical characteristics in the CAD cohort in Sapporo Medical University Hospital}

There are some differences between the characteristics of patients with CAD enrolled in Sapporo Medical University Hospital (see online supplementary table S2) and those in the BOREAS-CAG Registry. The patients in the Sapporo Medical University Hospital cohort showed lower eGFR $\left(53.1 \pm 24.1\right.$ vs $\left.64.0 \pm 35.0 \mathrm{~mL} / \mathrm{min} / 1.73 \mathrm{~m}^{2}\right)$ and a lower LDL cholesterol level $(91.2 \pm 33.3$ vs 110 $\pm 33.4 \mathrm{mg} / \mathrm{dL}$, online supplementary table S1 and S2). However, as was found for patients in the BOREAS-CAG Registry, levels of BNP and UA and the proportion of patients on a $\beta$ blocker were significantly higher in patients with $\mathrm{AF}$ than in those without $\mathrm{AF}$ (see online supplementary table S2). BNP and UA were also shown to be independently associated with the presence of $\mathrm{AF}$ 
by multivariate logistic regression analysis (see online supplementary table S3), reinforcing the results of the BOREAS-CAG Registry analysis.

\section{DISCUSSION}

The results of cross-sectional analyses of patients in the BOREAS-CAG Registry suggest that the major risk factors of $\mathrm{AF}$ are different in patients with CAD and those without CAD: high UA level in patients with CAD versus no statin use in patients without CAD, though high BNP level was a common risk factor of AF regardless of the presence or absence of CAD. The association between UA and AF in the patients with CAD was confirmed by analysis of a separate group of patients enrolled in our institute.

The findings in the analyses of the BOREAS-CAG Registry seem to be different to those in the classical AF literature. Clinically apparent, symptomatic CAD was shown to be an established risk factor for $\mathrm{AF}$ in previous studies, ${ }^{19}{ }^{20}$ whereas multivariate analysis in the present study showed that the prevalence of AF was lower in patients with CAD than in patients without CAD and that the absence of CAD was independently associated with AF (table 2). This discrepancy was unexpected, but there are two non-mutually exclusive explanations. First, criteria for enrolment of study participants in this study were different from those in previous observational studies in which general populations were enrolled. ${ }^{8}$ In the BOREAS-CAG Registry, patients who underwent coronary angiography for evaluation of chest pain, discomfort or other signs suggestive of CAD were consecutively enrolled. Thus, it is possible that AF was a major cause of non-CAD chest symptoms, resulting in a high prevalence of $\mathrm{AF}(13.2 \%)$ in patients without CAD. In fact, alteration in the CAD-AF association by selection of a specific group of patients was observed in the COMET study in which 3029 patients with heart failure (NYHA II-IV) were enrolled. ${ }^{21}$ In the COMET study, prevalence of angiographically confirmed CAD was significantly lower in patients with $\mathrm{AF}$ than in patients without $\mathrm{AF}$ (52.8\% vs $60.1 \%, \mathrm{p}=0.016$ ), apparently arguing against an association between $\mathrm{AF}$ and $\mathrm{CAD}$ in a general population. Second, difference in medication may be involved in the difference in AF prevalence between the patients with $\mathrm{CAD}$ and the patients without CAD. In the BOREAS-CAG Registry, a significantly smaller proportion of patients without CAD were administered a statin than the patients with CAD (see online supplementary table $\mathrm{S} 1$ ), and statin administration was independently associated with the absence of AF in the overall registry data (table 2). Thus, more frequent use of a statin in patients with CAD might outweigh the enhanced susceptibility to $\mathrm{AF}$ by the presence of CAD.

Meta-analysis of randomised controlled trials demonstrated that statin therapy was associated with a decreased risk of $\mathrm{AF}$ or recurrence of $\mathrm{AF}$ in patients in sinus rhythm with a history of previous AF, undergoing cardiac surgery or after acute coronary syndrome. ${ }^{22}$ The COMET study also showed that prevalence of statin administration was significantly higher in patients without $\mathrm{AF}$ than in those with $\mathrm{AF}(23.7 \%$ vs $10.5 \%$, $\mathrm{p}<0.0001) .{ }^{21}$ The beneficial effect of a statin seemed to be more pronounced in the prevention of $\mathrm{AF}$ recurrence than in primary prevention of $\mathrm{AF}^{22}{ }^{23}$ In this study, association of no statin use with AF was significant in the non-CAD group but not in the CAD group. Why statin use was not selected as a significant explanatory variable for $\mathrm{AF}$ in patients with $\mathrm{CAD}$ is unclear. When patients who were administered statin were analysed, LDL cholesterol level was significantly higher in the CAD group than in the non-CAD group $(108.5 \pm 35$ vs $98.1 \pm 35.0, p=0.003)$. This finding suggests that insufficient cholesterol-lowering effects of statins in patients with CAD may underlie the lack of statin-associated reduction in AF prevalence. However, this possibility is unlikely because of the following reasons. First, the level of LDL cholesterol tended to be lower in patients with $\mathrm{AF}$ than in those without AF in the non-CAD and CAD groups (table 3 ). Second, treatment with a statin was not associated with significant reduction of $\mathrm{AF}$ when the LDL cholesterol level was $<140 \mathrm{mg} / \mathrm{dL},<120 \mathrm{mg} / \mathrm{dL}$ or even $<100 \mathrm{mg} / \mathrm{dL}$ in the CAD group (see online supplementary figure S3), whereas prevalence of AF was consistently lower in statin-treated patients regardless of the LDL cholesterol level in the non-CAD group (see online supplementary figure S2).

Hyperuricemia has been shown to be associated with $\mathrm{AF}$ in patients with $\mathrm{HT}^{24}$ heart failure ${ }^{25}$ or type 2 diabetes mellitus. ${ }^{26}$ It has also been reported that serum UA level was significantly correlated with the development of $\mathrm{AF}$ in a concentration-dependent manner, ${ }^{27}$ and a meta-analysis of 6 cross-sectional and 3 cohort studies confirmed the association of hyperuricemia with $\mathrm{AF}^{28}$ However, to the best of our knowledge, the relationship between $\mathrm{CAD}$ and UA-associated AF risk has not been examined in detail. In this study, UA levels were similar in the $\mathrm{CAD}$ and non-CAD groups (see online supplementary table S1), but UA was selected as a significant explanatory variable of AF only in the CAD group (table 4). Why UA was not associated with AF in the non-CAD patients is unclear. The serum level of UA in the non-CAD patients $(5.9 \pm 1.7 \mathrm{mg} / \mathrm{dL})$ was slightly lower than levels in earlier studies showing UA-associated increased risk of $\mathrm{AF} \quad(6.2 \pm 0.2$ vs 5.3 $\pm 1.6 \mathrm{mg} / \mathrm{dL}, 6.4 \pm 2.1 \mathrm{vs} 5.7 \pm 1.9 \mathrm{mg} / \mathrm{dL}$ and $6.3 \pm 1.6$ vs 5.0 $\pm 1.3 \mathrm{mg} / \mathrm{dL}$ for patients with $\mathrm{AF}$ and those without $\mathrm{AF}$, respectively $\left.{ }^{24-26}\right)$. Besides, since the present analysis is cross-sectional, we cannot exclude the possibility that the duration of elevated UA level was shorter in participants of this study than in participants of earlier studies, in which patients free from AF at baseline were followed for 10 years. ${ }^{26}$ Another possible explanation is alteration in myocardial sensitivity to UA in the presence of CAD. However, there is no clinical evidence supporting this speculation. 
A significant association of high $\mathrm{UA}$ level with $\mathrm{AF}$ development and an association of statin use with reduced AF incidence shown in this study and earlier studies suggest possible benefits of UA-lowering agents and statins for prevention of AF. Emerging evidence suggests that oxidative stress and inflammation promote structural and electrical cardiac remodelling of the atrium, predisposing the heart to atrial fibrosis and $\mathrm{AF}^{29} 30$ Statins and allopurinol, a xanthine oxidase inhibitor to reduce production of UA, have been shown to have anti-inflammatory/antioxidative actions ${ }^{31-34}$ and possibly intervene in the process of atrial remodelling by risk factors of AF. However, the clinical benefit of statins or allopurinol in prevention of $\mathrm{AF}$ has not been unequivocally demonstrated. A recent meta-analysis demonstrated that perioperative statin therapy in statinnaive patients with sinus rhythm undergoing cardiac surgery was associated with a decrease in the development of postoperative $\mathrm{AF}^{35}$ In contrast, a recent randomised clinical trial with 1922 patients failed to show significant prevention of AF after cardiac surgery by rosuvastatin $(20 \mathrm{mg} /$ day $) .{ }^{36}$ Clinical benefit of allopurinol or other UA-lowering agents has not been critically tested by large randomised clinical trials, though allopurinol use was shown to be independently associated with a lower risk of new onset of AF in a cohort of elderly patients (78.2 \pm 7.2 years). ${ }^{37}$ Interestingly, longer duration of allopurinol administration achieved greater risk reduction, supporting the notion that suppression of atrial remodelling over a long period by the antioxidative property of the agent was involved in the clinical effects. ${ }^{37}$ Causal relationships between elevation of serum UA level, $\mathrm{AF}$ development and $\mathrm{CAD}$ remain to be examined in future studies.

Finally, since all patients in the BOREAS-CAG Registry were Japanese, the results are not directly translatable to other ethnicities. On the other hand, the Japanese are often significantly under-represented in clinical trials, and thus the results of the present analysis may be important in identifying trends in aetiology in such populations.

\section{STUDY LIMITATIONS}

There are several limitations in this study. First, as this was a cross-sectional study, the temporal order of variables cannot be determined, and thus the direction of causality is unclear. Second, AF was diagnosed if recorded clinically at the time of enrolment, as mentioned in the 'Methods' section and was not sought actively thereafter. Thus, PAF may have been missed in some patients. Third, information regarding types of statin was not obtained from the BOREAS-CAG Registry. Since the clinical benefit for AF may depend on the type of statin, ${ }^{23}$ we cannot exclude the possibility that types of statins were not well balanced in the patients with CAD and patients without $\mathrm{CAD}$, leading to the difference in the association between $\mathrm{AF}$ and statin use in the two groups. Finally, the present results are not directly applicable to the general population because all of the participants in this study had indications for coronary angiography. In fact, the prevalence of AF in the patients in the BOREAS-CAG Registry $(9.7 \%$ in overall patients, $6.1 \%$ in patients with CAD and $13.2 \%$ in patients without $\mathrm{CAD})$ was much higher than that in the general population (ie, 1-2\%). The high prevalences of HT $(69.0 \%)$, DM $(37.6 \%)$ and latent heart failure as indicated by elevated BNP may have predisposed patients in the BOREAS-CAG Registry to development of AF.

\section{CONCLUSIONS}

Cross-sectional analysis of the BOREAS-CAG Registry data indicated that risk factors of $\mathrm{AF}$ are distinct in patients with $\mathrm{CAD}$ and those without CAD. No statin administration was associated with $\mathrm{AF}$ in patients without $\mathrm{CAD}$, whereas high UA was the risk factor associated with $\mathrm{AF}$ in patients with $\mathrm{CAD}$. The present findings need to be confirmed by longitudinal studies.

Contributors NM and MT contribute to collection, analysis and interpretation of data and writing of manuscript. NK, JN and NN contribute to data collection and interpretation. $\mathrm{HO}, \mathrm{HA}$ and TaM contribute to statistical support and data interpretation. KT contributes to conception and design of study. TeM contributes to conception and design of study, data analysis and writing of the manuscript.

Funding This study was supported by the Sapporo Medical University Education and Research Grants.

Competing interests None declared.

Patient consent Obtained.

Ethics approval Clinical Investigation Ethics Committee of Sapporo Medical University Hospital.

Provenance and peer review Not commissioned; externally peer reviewed.

Data sharing statement No additional data are available.

Open Access This is an Open Access article distributed in accordance with the Creative Commons Attribution Non Commercial (CC BY-NC 4.0) license, which permits others to distribute, remix, adapt, build upon this work noncommercially, and license their derivative works on different terms, provided the original work is properly cited and the use is non-commercial. See: http:// creativecommons.org/licenses/by-nc/4.0/

\section{REFERENCES}

1. Chugh SS, Havmoeller R, Narayanan K, et al. Worldwide epidemiology of atrial fibrillation: a Global Burden of Disease 2010 Study. Circulation 2014;129:837-47.

2. Wolf PA, Abbott RD, Kannel WB. Atrial fibrillation as an independent risk factor for stroke: the Framingham Study. Stroke 1991;22:983-8.

3. Benjamin EJ, Wolf PA, D'Agostino RB, et al. Impact of atrial fibrillation on the risk of death: the Framingham Heart Study. Circulation 1998;98:946-52.

4. Bhatia RS, Tu JV, Lee DS, et al. Outcome of heart failure with preserved ejection fraction in a population-based study. $N$ Engl J Med 2006;355:260-9.

5. Hamaguchi S, Yokoshiki H, Kinugawa S, et al. Effects of atrial fibrillation on long-term outcomes in patients hospitalized for heart failure in Japan: a report from the Japanese Cardiac Registry of Heart Failure in Cardiology (JCARE-CARD). Circ $J$ 2009;73:2084-90.

6. Kirchhof P, Lip GY, Van Gelder IC, et al. Comprehensive risk reduction in patients with atrial fibrillation: emerging diagnostic and therapeutic options-a report from the 3rd 
Atrial Fibrillation Competence NETwork/European Heart Rhythm Association consensus conference. Europace 2012:14:8-27.

7. Rodriguez F, Stefanick ML, Greenland P, et al. Racial and ethnic differences in atrial fibrillation risk factors and predictors in women: findings from the Women's Health Initiative. Am Heart $J$ 2016;176:70-7.

8. Schnabel RB, Sullivan LM, Levy $\mathrm{D}$, et al. Development of a risk score for atrial fibrillation (Framingham Heart Study): a community-based cohort study. Lancet 2009;373:739-45.

9. Alonso A, Krijthe BP, Aspelund T, et al. Simple risk model predicts incidence of atrial fibrillation in a racially and geographically diverse population: the CHARGE-AF consortium. J Am Heart Assoc 2013;2: e000102.

10. Chamberlain AM, Agarwal SK, Folsom AR, et al. A clinical risk score for atrial fibrillation in a biracial prospective cohort (from the Atherosclerosis Risk in Communities [ARIC] study). Am J Cardiol 2011;107:85-91.

11. Chua SK, Shyu KG, Lu MJ, et al. Renal dysfunction and the risk of postoperative atrial fibrillation after cardiac surgery: role beyond the CHA2DS2-VASc score. Europace 2015;17:1363-70.

12. van Oosten EM, Hamilton A, Petsikas D, et al. Effect of preoperative obstructive sleep apnea on the frequency of atria fibrillation after coronary artery bypass grafting. Am J Cardiol 2014;113:919-23.

13. Shimony A, Afilalo J, Flynn AW, et al. Usefulness of right ventricular dysfunction to predict new-onset atrial fibrillation following coronary artery bypass grafting. Am J Cardiol 2014;113:913-18.

14. Flaker GC, Belew K, Beckman K, et al. Asymptomatic atrial fibrillation: demographic features and prognostic information from the Atrial Fibrillation Follow-up Investigation of Rhythm Management (AFFIRM) study. Am Heart J 2005;149:657-63.

15. Lip GY, Beevers DG. ABC of atrial fibrillation. History, epidemiology, and importance of atrial fibrillation. BMJ 1995;311:

1361-3.

16. Pedersen OD, Abildstrom SZ, Ottesen MM, et al. Increased risk of sudden and non-sudden cardiovascular death in patients with atrial fibrillation/flutter following acute myocardial infarction. Eur Heart $J$ 2006;27:290-5.

17. Suttorp MJ, Kingma JH, Koomen EM, et al. Recurrence of paroxysmal atrial fibrillation or flutter after successful cardioversion in patients with normal left ventricular function. Am J Cardiol 1993;71:710-13.

18. Corley SD, Epstein AE, DiMarco JP, et al. Relationships between sinus rhythm, treatment, and survival in the Atrial Fibrillation Follow-Up Investigation of Rhythm Management (AFFIRM) Study. Circulation 2004;109:1509-13.

19. Benjamin EJ, Levy D, Vaziri SM, et al. Independent risk factors for atrial fibrillation in a population-based cohort. The Framingham Heart Study. JAMA 1994;271:840-4.

20. Krahn AD, Manfreda J, Tate RB, et al. The natural history of atrial fibrillation: incidence, risk factors, and prognosis in the Manitoba Follow-Up Study. Am J Med 1995;98:476-84.
21. Swedberg K, Olsson LG, Charlesworth A, et al. Prognostic relevance of atrial fibrillation in patients with chronic heart failure on long-term treatment with beta-blockers: results from COMET. Eur Heart J 2005;26:1303-8.

22. Fauchier L, Pierre B, de Labriolle A, et al. Antiarrhythmic effect of statin therapy and atrial fibrillation a meta-analysis of randomized controlled trials. J Am Coll Cardiol 2008;51:828-35.

23. Fang WT, Li HJ, Zhang $\mathrm{H}$, et al. The role of statin therapy in the prevention of atrial fibrillation: a meta-analysis of randomized controlled trials. Br J Clin Pharmacol 2012;74:744-56.

24. Liu $\mathrm{T}$, Zhang $\mathrm{X}$, Korantzopoulos $\mathrm{P}$, et al. Uric acid levels and atrial fibrillation in hypertensive patients. Intern Med 2011;50:799-803.

25. Tekin G, Tekin YK, Erbay AR, et al. Serum uric acid levels are associated with atrial fibrillation in patients with ischemic heart failure. Angiology 2013;64:300-3.

26. Valbusa F, Bertolini L, Bonapace S, et al. Relation of elevated serum uric acid levels to incidence of atrial fibrillation in patients with type 2 diabetes mellitus. Am J Cardiol 2013;112:499-504.

27. Tamariz L, Agarwal S, Soliman EZ, et al. Association of serum uric acid with incident atrial fibrillation (from the Atherosclerosis Risk in Communities [ARIC] study). Am J Cardiol 2011;108:1272-6.

28. Tamariz L, Hernandez F, Bush A, et al. Association between serum uric acid and atrial fibrillation: a systematic review and meta-analysis. Heart Rhythm 2014;11:1102-8.

29. Nattel S, Burstein B, Dobrev D. Atrial remodeling and atrial fibrillation: mechanisms and implications. Circ Arrhythm Electrophysiol 2008;1:62-73.

30. Youn JY, Zhang J, Zhang Y, et al. Oxidative stress in atrial fibrillation: an emerging role of NADPH oxidase. $J$ Mol Cell Cardiol 2013;62:72-9.

31. Kumar AP, Reynolds WF. Statins downregulate myeloperoxidase gene expression in macrophages. Biochem Biophys Res Commun 2005;331:442-51

32. Reilly SN, Jayaram R, Nahar K, et al. Atrial sources of reactive oxygen species vary with the duration and substrate of atrial fibrillation: implications for the antiarrhythmic effect of statins. Circulation 2011:124:1107-17.

33. Givertz MM, Mann DL, Lee KL, et al. Xanthine oxidase inhibition for hyperuricemic heart failure patients: design and rationale of the EXACT-HF study. Circ Heart Fail 2013;6:862-8.

34. George J, Carr E, Davies J, et al. High-dose allopurinol improves endothelial function by profoundly reducing vascular oxidative stress and not by lowering uric acid. Circulation 2006;114:2508-16.

35. Rezaei Y, Gholami-Fesharaki M, Dehghani MR, et al. Statin antiarrhythmic effect on atrial fibrillation in statin-naive patients undergoing cardiac surgery: a meta-analysis of randomized controlled trials. J Cardiovasc Pharmacol Ther 2016;21:167-76.

36. Zheng Z, Jayaram R, Jiang L, et al. Perioperative rosuvastatin in cardiac surgery. N Engl J Med 2016;374:1744-53.

37. Singh JA, Yu S. Allopurinol and the risk of atrial fibrillation in the elderly: a study using Medicare data. Ann Rheum Dis 2017; 76:72-8 